\title{
Continuous prostate-specific antigen rise despite salvage radiotherapy following radical prostatectomy: Pattern of clinical relapse and predictive factors
}

\author{
CARSTEN NIEDER ${ }^{1,2}$, KIRSTEN MARIENHAGEN ${ }^{3}$, ROY M. KRISTENSEN ${ }^{4}$, \\ TORBJØRN SØRBYE ${ }^{4}$ and LARS HOEM ${ }^{4}$ \\ ${ }^{1}$ Department of Oncology and Palliative Medicine, Nordland Hospital, Bodø 8092; \\ ${ }^{2}$ Institute of Clinical Medicine, Faculty of Health Sciences, University of Troms $\varnothing$, Troms $\varnothing 6038$; \\ ${ }^{3}$ Department of Oncology, University Hospital of North Norway, Troms $\varnothing 9038$; \\ ${ }^{4}$ Department of Urology, Nordland Hospital, Bodø 8092, Norway
}

Received November 27, 2014; Accepted November 10, 2015

DOI: $10.3892 / \mathrm{ol} .2015 .4005$

\begin{abstract}
Salvage radiotherapy for post-prostatectomy biochemical recurrence does not always control the disease. It would be useful to identify patients who would not benefit from radiotherapy to the prostate bed prior to making treatment recommendations. One such group of patients is those who experience continuously rising prostate-specific antigen (PSA) despite radiotherapy. The purpose of this study was to identify risk factors for continuous PSA increase and the pattern of radiological relapse during follow-up. We performed a retrospective comparison of two patient groups with PSA decline or continuous increase following salvage radiotherapy to the prostate bed. All patients received 3-D conformal radiotherapy ( 35 fractions of 2 Gy). Patients with continuous PSA increase were more likely to have had complete surgical resection (negative margins) and a shorter interval to radiotherapy ( $<24$ months). However, the only statistically significant risk factor was Gleason score. Sixty-four percent of patients with a Gleason score of 9 developed continuously increasing PSA, indicating that residual subclinical cancer was not located in the prostate bed. The median time to radiological recurrence was 43 months. Isolated pelvic nodal recurrence was uncommon. Almost all patients with radiological recurrence had high-risk disease, in particular stage pT3. In conclusion, the majority of patients with biologically aggressive tumors with Gleason score 9 were not adequately treated with prostate bed radiotherapy alone. The predominant pattern of radiological
\end{abstract}

Correspondence to: Professor Carsten Nieder, Department of Oncology and Palliative Medicine, Nordland Hospital, 164 Prinsensgate, Bodø 8092, Norway

E-mail: carsten.nieder@nlsh.no

Key words: radiation oncology, radiotherapy, prostate cancer, prostate-specific antigen, salvage treatment recurrence was outside of the pelvis. Therefore, the problem of distant micrometastases has to be addressed.

\section{Introduction}

Not all patients with organ-confined prostate cancer treated with radical prostatectomy experience long-term disease control. Increasing levels of prostate-specific antigen (PSA) following surgery due to biochemical relapse (BCR) typically precede the development of clinical recurrence. In the setting of BCR without detectable distant metastases, salvage radiotherapy to the prostate bed is an established therapeutic option (1-6), based on the assumption that all residual cancer cells are located there. Two different immediate results might be observed: success, as indicated by PSA decline (possibly followed by relapse during follow-up), or failure, as indicated by unaffected PSA progression. In the case of immediate failure (or a continuous increase in PSA), the hypothesis is proved incorrect: in these patients, the residual cancer cells are not located in the prostate bed. The imaging methods currently in use are unable to detect microscopic cancer deposits. Therefore, optimal patient selection needs to be based on other approaches. Pre-treatment identification of all patients who are likely to develop continuously rising PSA despite radiotherapy to the prostate bed would be desirable as these patients could be considered for other therapies. The purpose of this study was to analyze predictive factors for continuous PSA increase, and to describe the pattern of radiologically detected recurrences during follow-up. We perform a retrospective comparison of two patient groups with PSA decline or continuous increase following salvage radiotherapy to the prostate bed.

\section{Patients and methods}

This retrospective analysis included 83 males with prostate cancer treated with radical prostatectomy who had persistently elevated PSA or who developed BCR, i.e. PSA $>0.2 \mathrm{ng} / \mathrm{ml}$, after non-detectable PSA immediately after surgery. The 
patients were treated with 3-D conformal salvage radiotherapy at the University Hospital in Troms $\varnothing$ or the affiliated academic teaching hospital Nordland Hospital in Bodø, Norway. The patients were treated between 2006 and 2014. The study was performed as a retrospective analysis of salvage radiotherapy. As a quality of care analysis, no approval from the Regional Committee for Medical and Health Research Ethics (REK) was necessary. None of the patients had clinical or radiological evidence of macroscopic disease following isotope bone scan, computed tomography (CT) of the pelvis and abdomen, and magnetic resonance imaging of the pelvis. Positron-emission tomography (PET)-CT was not used. Patients with node-positive disease at the time of surgery or re-staging were not included. In addition to N0 stage, all patients were required to have had a maximum PSA of $2.0 \mathrm{ng} / \mathrm{ml}$ at the time of salvage radiotherapy and to be endocrine treatment-naïve. The patient characteristics are shown in Table I. The dose was prescribed according to the the ICRU 50 guidelines. The $95 \%$ isodose line encompassed the planning target volume (PTV) and the maximum dose did not exceed $107 \%$ of the prescribed dose. The dose per fraction was $2 \mathrm{~Gy}$, and the total dose was $70 \mathrm{~Gy}$. CT scans were used to define the clinical target volume (CTV) based on histological and surgical reports. The prostate bed was contoured and in case of seminal vesicle involvement the CTV was expanded to include this area. None of the patients received treatment to the pelvic lymph nodes. The margins added to the CTV to create the PTV were $1 \mathrm{~cm}$ in all directions. All patients were treated with 6-15 MV photons from a linear accelerator via 4 to 6 individually shaped treatment fields.

Biochemical relapse-free survival (BRFS) and further treatment. A biochemical recurrence following salvage radiotherapy was defined as a PSA value above $0.2 \mathrm{ng} / \mathrm{ml}$. PSA was assessed every 3 months following radiotherapy. We defined continuous rise as at least two subsequent PSA increases from the pre-radiotherapy level. Re-staging following radiotherapy included the same imaging modalities as mentioned above; i.e. no PET-CT. The timing of imaging and additional treatment following BCR was at the discretion of the patient's urologist. No uniform criteria for any intervention were applied.

Statistical analysis. The Chi-square test and Fisher's exact test, where applicable, were employed for comparison of dichotomous variables, and the Mann-Whitney U test was employed for continuous variables. The Kaplan-Meier method was employed for estimates of BRFS and metastasis-free survival from the initiation of salvage radiotherapy. $\mathrm{P}<0.05$ was considered to indicate a statistically significant difference. All tests were two-sided.

\section{Results}

Of the 83 patients, 21 developed continuously rising PSA. The others responded biochemically (51 completely, i.e. PSA $<0.2 \mathrm{ng} / \mathrm{ml}$, and 11 partially, i.e. a variable degree of PSA decline). There was a trend towards a shorter interval between surgery and radiotherapy in patients with continuous PSA increase ( $\mathrm{P}=0.07$ if the cut-off was set to $<24$ vs. $\geq 24$ months). There was also a trend towards higher rates of $\mathrm{R} 0$ resection,

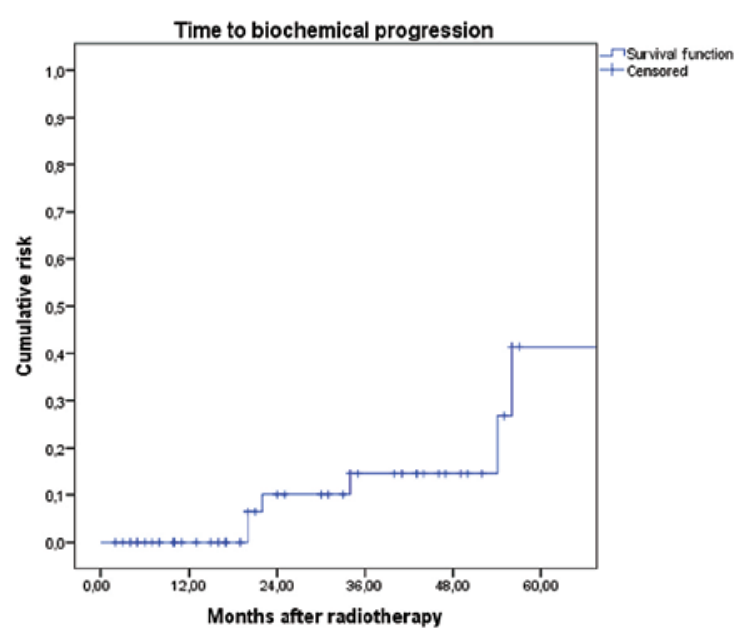

Figure 1. Actuarial biochemical relapse-free survival in 51 patients with optimal initial response to prostate bed radiotherapy, i.e. prostate-specific antigen nadir $<0.2 \mathrm{ng} / \mathrm{ml}$. Patients without relapse $(\mathrm{n}=46)$ had a median follow-up of 21 months.

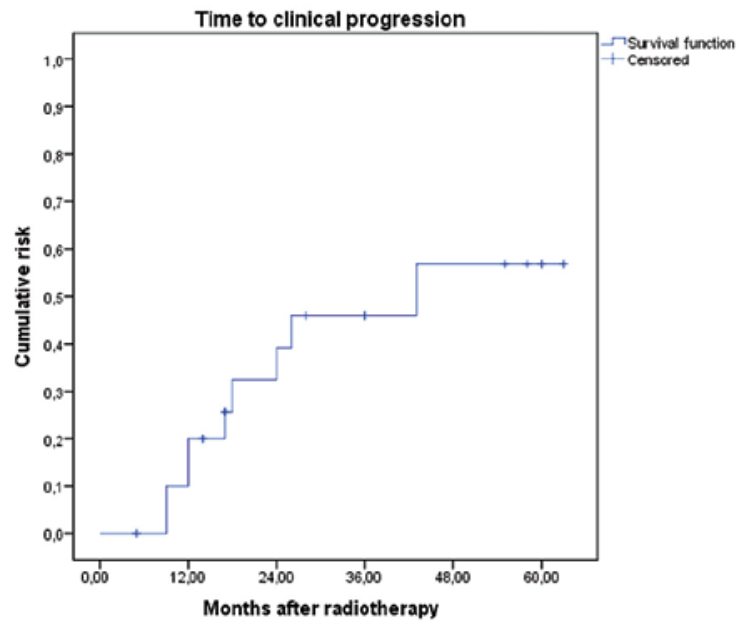

Figure 2. Actuarial time to radiological recurrence in 21 patients with continuous prostate-specific antigen increase following prostate bed radiotherapy. Median time to radiological recurrence was 43 months. Median follow-up in patients without detectable macroscopic recurrence $(n=12)$ was 32 months.

i.e. no microscopically involved margins, in patients with continuous PSA increase $(\mathrm{P}=0.066)$. Thirty-six percent of patients with R0 resection developed continuously rising PSA, compared with $17 \%$ of patients with involved margins. Sixty-four percent of patients with Gleason score 9 developed continuously rising PSA. The Gleason score was the only statistically significant risk factor $(\mathrm{P}=0.007)$. Of the five patients with Gleason score 9 and perineural invasion, four developed continuously rising PSA. Neither pre- nor post-surgical risk classification (low, intermediate or high) were significantly associated with the risk of continuous PSA increase. Neither the diagnosis of pT3b stage nor simultaneous presence of two high-risk features was a significant risk factor.

Table II shows the outcome parameters in the groups with different PSA responses. Figure 1 shows actuarial BRFS in patients with optimal initial response, i.e. PSA nadir 
Table I. Patient characteristics.

\begin{tabular}{|c|c|c|c|}
\hline Parameter & $\begin{array}{l}\text { Continuous PSA increase } \\
\qquad(\mathrm{n}=21)\end{array}$ & $\begin{array}{l}\text { PSA decline } \\
\qquad(\mathrm{n}=62)\end{array}$ & $\begin{array}{l}\text { Significance level } \\
\quad(\text { if } \mathrm{P}<0.2)\end{array}$ \\
\hline Median age, years (range) & $64(57-70)$ & $64(51-71)$ & \\
\hline Median PSA (ng/ml) before surgery & $8.8(3.1-19.0)$ & $9.0(2.8-35.0)$ & \\
\hline Median PSA before RT (range) & $0.6(0.3-1.8)$ & $0.5(0.2-2.0)$ & 0.176 \\
\hline Median interval to RT, months & $16(3-86)$ & $25(4-101)$ & 0.195 \\
\hline PSA detected cancer & 12 & 35 & \\
\hline Clinically symptomatic cancer & 9 & 27 & \\
\hline Pre-surgery low-risk disease ${ }^{a}$ & 2 & 11 & \\
\hline Pre-surgery intermediate risk disease & 13 & 40 & \\
\hline Pre-surgery high-risk disease & 5 (1 unknown) & 11 & \\
\hline Gleason score difference ${ }^{b}$ & 10 & 33 & \\
\hline No Gleason score difference & 11 & 29 & \\
\hline Perineural infiltration & 13 & 38 & \\
\hline No perineural infiltration & 8 & 24 & \\
\hline Involved margin (R1) & 8 & 40 & \\
\hline Clear margin (R0) & 12 (1 unknown) & 21 (1 unknown) & 0.066 \\
\hline Post-surgery Gleason score $3+3$ & 1 & 10 & \\
\hline Post-surgery Gleason score 3+4 & 6 & 29 & \\
\hline Post-surgery Gleason score $4+3$ & 6 & 10 & \\
\hline Post-surgery Gleason score $3+5$ & 0 & 2 & \\
\hline Post-surgery Gleason score 4+4 & 1 & 5 & \\
\hline Post-surgery Gleason score 9 & 7 & 4 (2 unknown) & 0.007 \\
\hline Post-surgery T stage 2 & 11 & 32 & \\
\hline Post-surgery $\mathrm{T}$ stage $3 \mathrm{a}$ & 5 & 23 & \\
\hline Post-surgery T stage $3 \mathrm{~b}$ & 4 (1 unknown) & 7 & \\
\hline Lymph node dissection $^{c}$ & 7 & 19 & \\
\hline No lymph node dissection & 14 & 43 & \\
\hline Charlson comorbidity index 0 & 12 & 34 & \\
\hline Comorbidity present $^{\mathrm{d}}$ & 8 (1 unknown) & 28 & \\
\hline
\end{tabular}

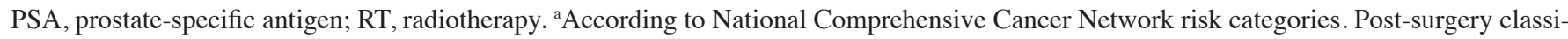
fication was not significant either. ${ }^{b}$ Upgrade from biopsy to prostatectomy histology. ${ }^{c}$ Median number of pathologically examined pelvic nodes was 7 in both groups. ${ }^{\mathrm{d}}$ No significant impact of specific comorbidities including diabetes mellitus and other metachronous tumors. Smoking status was not significant either.

Table II. Prostate-specific antigen outcome during follow-up.

\begin{tabular}{lccc}
\hline Parameter & $\begin{array}{c}\text { Continuous PSA } \\
\text { increase }(\mathrm{n}=21)\end{array}$ & $\begin{array}{c}\text { PSA decline to nadir } \\
<0.2 \mathrm{ng} / \mathrm{ml}(\mathrm{n}=51)\end{array}$ & $\begin{array}{l}\text { PSA decline to nadir } \\
\geq 0.2 \mathrm{ng} / \mathrm{ml}(\mathrm{n}=11)\end{array}$ \\
\hline $\begin{array}{l}\text { New biochemical relapse } \\
\text { following salvage RT }\end{array}$ & 21, immediately after RT & 5, during follow-up (Fig. 1) & 8, during follow-up \\
\hline
\end{tabular}

PSA, prostate-specific antigen; RT, radiotherapy.

$<0.2 \mathrm{ng} / \mathrm{ml}$. Patients without PSA relapse had a median follow-up of 21 months. Figure 2 shows the actuarial time to radiological recurrence in patients with continuous PSA increase. A total of nine recurrences were detected (three in pelvic nodes only, three in pelvic and para-aortic nodes simultaneously, and three cases with bone metastases), and one patient succumbed
23 months after the diagnosis of bone metastases. Isolated pelvic nodal recurrence was uncommon. The median time to radiological recurrence was 43 months. The median follow-up time in patients without detectable macroscopic recurrence was 32 months. Eight of the nine patients with radiological recurrence had high-risk disease (all stage pT3, four with Gleason 
score 9). The remaining patient (isolated pelvic nodal relapse) had initial PSA $19 \mathrm{ng} / \mathrm{ml}$, stage pT2c and Gleason score 3+4. Three patients were already on endocrine treatment when radiological relapse was detected, and the others started afterwards. Eight of the 12 patients without detectable macroscopic recurrence started endocrine treatment during follow-up.

\section{Discussion}

The purpose of this study was to analyze predictive factors for continuous PSA increase following salvage radiotherapy to the prostate bed, and to describe the pattern of radiologically detected recurrence during follow-up. We selected this endpoint as patients whose biochemical disease progression remains unaffected by radiotherapy to the prostate bed represent a group that does not benefit from such treatment and rather requires other approaches. Of course, there is also a certain risk of disease progression in patients with initial PSA response (7-10). However, previous studies suggest that the time interval between post-radiotherapy BCR and overt metastatic disease is often so long that life expectancy is not affected $(11,12)$. Our patients with continuous PSA increase had a median age of 64 years and a median interval to radiological recurrence of 43 months. Together with the fact that most recurrences were located outside of the pelvis (M1 stage), these results indicate the life-threatening character of continuously rising PSA.

When interpreting the results, certain potential limitations of the study should be noted, e.g. that retrospective studies typically provide indications and hypotheses rather than definitive conclusions.

Unfortunately, risk factors for continuously rising PSA were difficult to identify. With only 21 events, the statistical power of this study was limited. For this reason, we refrained from multivariate analysis. Another weak point was the limited follow-up time following radiotherapy. The only statistically significant risk factor was high Gleason score, and there was also a trend for negative surgical margins. In exploratory analyses, the highest risk was observed in patients with a Gleason score of 9 and perineural invasion (80\%). However, this finding should be interpreted with caution as it was derived from an extremely small subgroup of five patients. In a comparably small study ( $\mathrm{n}=61 \mathrm{vs} .83$ in the present analysis), different results were obtained (13). In addition to negative surgical margins, PSA velocity $\geq 0.8 \mathrm{ng} / \mathrm{ml} /$ year was associated with higher risk of persistent PSA increase, indicating non-response to salvage radiotherapy. The Gleason score was not statistically significant. In our study, PSA velocity was unknown. If patients with delayed PSA relapse following salvage radiotherapy were to be included, as was typically the case in previous analyses, the interpretation of results becomes more complicated. Primary non-response is associated with an absence of cancer cells in the prostate bed. This could also be termed a 'staging error' since current imaging is not able to detect microscopic cancer deposits. If PSA relapse develops later during follow-up, reasons including surviving cancer cells in the prostate bed need to be considered in addition to out-of-field relapse. Survival may result from geographical miss or a more general, insufficiently low radiation dose.

Possible ways forward for patients with a high risk of continuous PSA increase following prostate bed radiotherapy alone include more effective pre-radiotherapy staging, for example with PET-CT, which has been demonstrated to impact patient management in this setting (14-17). Immediate postoperative radiotherapy could also be helpful (18-20), since it is able to kill residual cancer cells as early as possible, thereby preventing metastatic seeding from the prostate bed during the time interval that elapses between surgery and salvage radiotherapy. Salvage extended-field pelvic radiotherapy alone may not be sufficient, since most of our patients had radiological relapses outside of this region. The addition of systemic therapy appears to be necessary to address the issue of distant micrometastases, e.g. endocrine, cytotoxic or immune therapy (21-25).

The majority of patients with biologically aggressive tumors with Gleason score 9 were not adequately treated with prostate bed radiotherapy alone. The predominant pattern of radiological recurrence was outside of the pelvis. Therefore, the problem of distant micrometastases has to be addressed.

\section{References}

1. De Meerleer G, Fonteyne V, Meersschout S, et al: Salvage intensity-modulated radiotherapy for rising PSA after radical prostatectomy. Radiother Oncol 89: 205-213, 2008.

2. Bernard JR Jr, Buskirk SJ, Heckman MG, et al: Salvage radiotherapy for rising prostate-specific antigen levels after radical prostatectomy for prostate cancer: dose-response analysis. Int J Radiat Oncol Biol Phys 76: 735-740, 2010.

3. Trock BJ, Han M, Freedland SJ, et al: Prostate cancer-specific survival following salvage radiotherapy vs observation in men with biochemical recurrence after radical prostatectomy. JAMA 299: 2760-2769, 2008.

4. Jereczek-Fossa BA, Zerini D, Vavassori A, et al: Sooner or later? Outcome analysis of 431 prostate cancer patients treated with postoperative or salvage radiotherapy. Int J Radiat Oncol Biol Phys 74: 115-125, 2009.

5. Valicenti RK, Thompson I Jr, Albertsen P, et al: Adjuvant and salvage radiation therapy after prostatectomy: American Society for Radiation Oncology/American Urological Association guidelines. Int J Radiat Oncol Biol Phys 86: 822-888, 2013.

6. Shelan M, Abo-Madyan Y, Welzel G, et al: Dose-escalated salvage radiotherapy after radical prostatectomy in high risk prostate cancer patients without hormone therapy: outcome, prognostic factors and late toxicity. Radiat Oncol 8: 276, 2013.

7. Stephenson AJ, Scardino PT, Kattan MW, et al: Predicting the outcome of salvage radiation therapy for recurrent prostate cancer after radical prostatectomy. J Clin Oncol 25: 2035-2041, 2007.

8. Macdonald OK, D'Amico AV, Sadetsky N, Shrieve DC and Carroll PR: Predicting PSA failure following salvage radiotherapy for a rising PSA post-prostatectomy: from the CaPSURE database. Urol Oncol 26: 271-275, 2008

9. Moreira DM, Jayachandran J, Presti JC Jr, et al: Validation of a nomogram to predict disease progression following salvage radiotherapy after radical prostatectomy: results from the SEARCH database. BJU Int 104: 1452-1456, 2009.

10. Lohm G, Lütcke J, Jamil B, et al: Salvage radiotherapy in patients with prostate cancer and biochemical relapse after radical prostatectomy: long-term follow-up of a single-center survey. Strahlenther Onkol 190: 727-731, 2014.

11. Geinitz H, Riegel MG, Thamm R, et al: Outcome after conformal salvage radiotherapy in patients with rising prostate-specific antigen levels after radical prostatectomy. Int J Radiat Oncol Biol Phys 82: 1930-1937, 2012.

12. Wiegel T, Lohm G, Bottke D, et al: Achieving an undetectable PSA after radiotherapy for biochemical progression after radical prostatectomy is an independent predictor of biochemical outcome-results of a retrospective study. Int J Radiat Oncol Biol Phys 73: 1009-1016, 2009.

13. Miyake M, Tanaka N, Asakawa I, et al: Proposed salvage treatment strategy for biochemical failure after radical prostatectomy in patients with prostate cancer: a retrospective study. Radiat Oncol 9: 208, 2014. 
14. Ceci F, Herrmann K, Castellucci P, et al: Impact of (11) C-choline PET/CT on clinical decision making in recurrent prostate cancer: results from a retrospective two-centre trial. Eur J Nucl Med Mol Imaging 41: 2222-2231, 2014.

15. D'Angelillo RM, Sciuto R, Ramella S, et al: ${ }^{18} \mathrm{~F}$-choline positron emission tomography/computed tomography-driven high-dose salvage radiation therapy in patients with biochemical progression after radical prostatectomy: feasibility study in 60 patients. Int J Radiat Oncol Biol Phys 90: 296-302, 2014.

16. Rischke HC, Schäfer AO, Nestle U, et al: Detection of loca recurrent prostate cancer after radical prostatectomy in terms of salvage radiotherapy using dynamic contrast enhanced-MRI without endorectal coil. Radiat Oncol 7: 185, 2012.

17. Beresford MJ, Gillatt D, Benson RJ and Ajithkumar T: A systematic review of the role of imaging before salvage radiotherapy for post-prostatectomy biochemical recurrence. Clin Oncol (R Coll Radiol) 22: 46-55, 2010.

18. Bolla M, van Poppel H, Collette L, et al: Postoperative radiotherapy after radical prostatectomy: a randomised controlled trial (EORTC trial 22911). Lancet 366: 572-578, 2005.

19. Thompson IM Jr, Tangen CM, Paradelo J, et al: Adjuvant radiotherapy for pathologically advanced prostate cancer: a randomized clinical trial. JAMA 296: 2329-2335, 2006.

20. Wiegel T, Bottke D, Steiner U, et al: Phase III postoperative adjuvant radiotherapy after radical prostatectomy compared with radical prostatectomy alone in $\mathrm{pT} 3$ prostate cancer with postoperative undetectable prostate-specific antigen: ARO 96-02/AUO AP 09/95. J Clin Oncol 27: 2924-2930, 2009.
21. Choo R, Danjoux C, Gardner S, et al: Efficacy of salvage radiotherapy plus 2-year androgen suppression for postradical prostatectomy patients with PSA relapse. Int J Radiat Oncol Biol Phys 75: 983-989, 2009.

22. Pai HH, Eldridge B, Bishop D, et al: Does neoadjuvant hormone therapy improve outcome in prostate cancer patients receiving radiotherapy after radical prostatectomy? Can J Urol 16: 4541-4552, 2009.

23. Leung CM, Li SC, Chen TW, et al: Comprehensive microRNA profiling of prostate cancer cells after ionizing radiation treatment. Oncol Rep 31: 1067-1078, 2014.

24. Guttilla A, Bortolus R, Giannarini G, et al: Multimodal treatment for high-risk prostate cancer with high-dose intensity-modulated radiation therapy preceded or not by radical prostatectomy, concurrent intensified-dose docetaxel and long-term androgen deprivation therapy: results of a prospective phase II trial. Radiat Oncol 9: 24, 2014.

25. Grenader T, Plotkin Y, Gips M, Cherny N and Gabizon A: Diethylstilbestrol for the treatment of patients with castration-resistant prostate cancer: Retrospective analysis of a single institution experience. Oncol Rep 31: 428-434, 2014. 